Document downloaded from:

http://hdl.handle.net/10251/47877

This paper must be cited as:

Sanguesa, JA.; Fogue, M.; Garrido, P.; Martinez, FJ.; Cano Escribá, JC.; Tavares De Araujo Cesariny Calafate, CM. (2014). Using topology and neighbor information to overcome adverse vehicle density conditions. Transportation Research Part C: Emerging Technologies. 42:1-13. doi:10.1016/j.trc.2014.02.010.

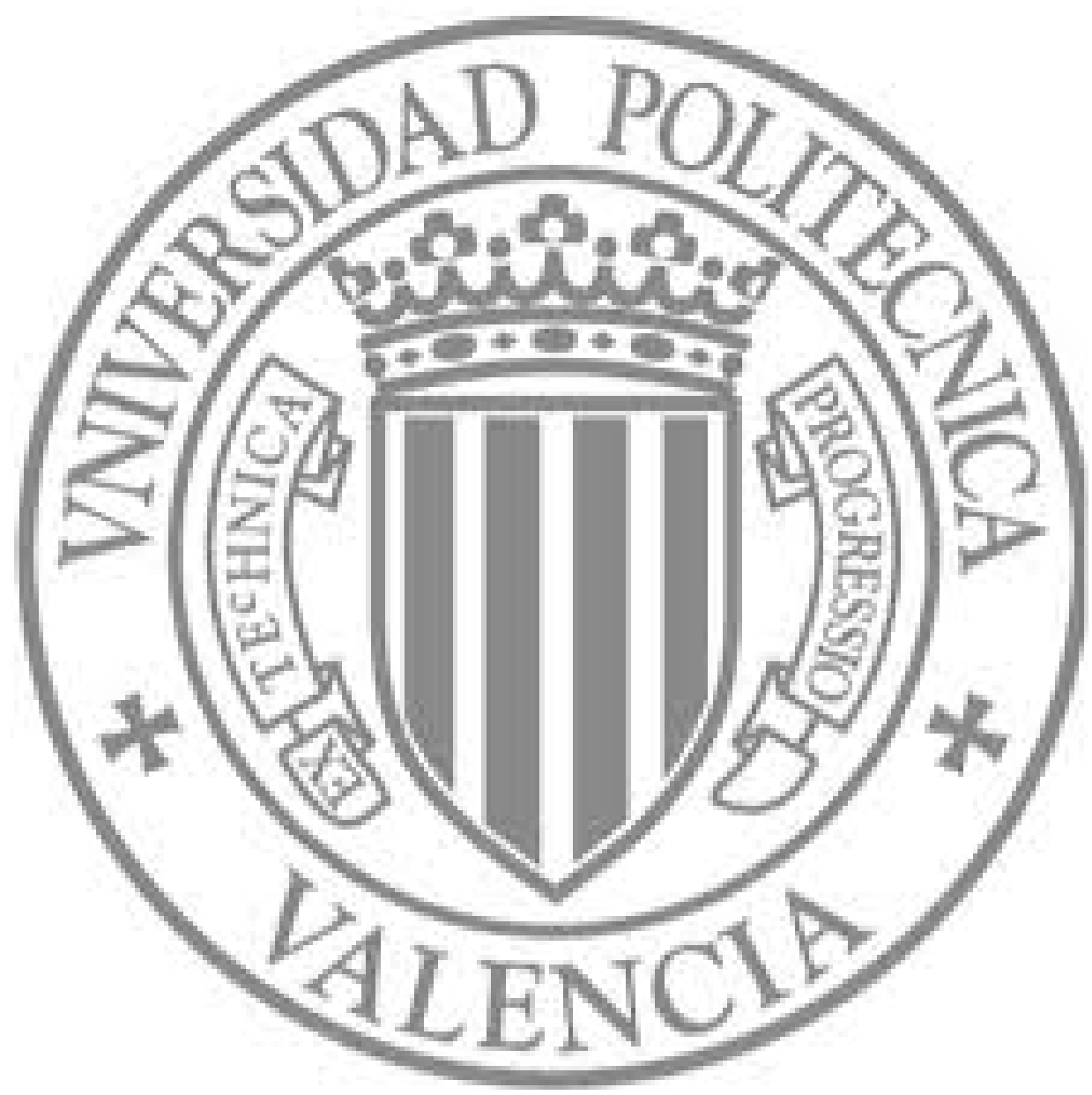

The final publication is available at

http://dx.doi.org/10.1016/j.trc.2014.02.010

Copyright Elsevier 


\title{
Using Topology and Neighbor Information to Overcome Adverse Vehicle Density Conditions
}

\author{
Julio A. Sanguesa, Manuel Fogue, Piedad Garrido, Francisco J. Martinez \\ University of Zaragoza, Spain \\ Email: \{jsanguesa,mfogue, piedad,f.martinez\}@unizar.es \\ Juan-Carlos Cano, Carlos T. Calafate \\ Universitat Politècnica de València, Spain \\ Email:\{jucano,calafate\}@disca.upv.es
}

\begin{abstract}
Vehicular networks supporting cooperative driving on the road have attracted much attention due to the plethora of new possibilities they offer to modern Intelligent Transportation Systems. However, research works regarding vehicular networks usually obviate assessing their proposals in scenarios including adverse vehicle densities far from the average values, despite being common in real urban environments. In this paper, we study the effect of these hostile conditions on the performance of different schemes providing warning message dissemination. The goal of these schemes is to maximize message delivery effectiveness, something difficult to achieve in adverse density scenarios. In addition, we propose the Neighbor Store and Forward (NSF) scheme, designed to be used under low density conditions, and the Nearest Junction Located (NJL) scheme, specially developed for high density conditions. Simulation results demonstrate that our proposals are able to outperform existing warning message dissemination schemes in urban environments under adverse vehicle density conditions. In particular, NSF reduces the warning notification time in low vehicle density scenarios, while increasing up to $23.3 \%$ the percentage of informed vehicles. As for high vehicle density conditions, NJL is able to inform the same percentage of vehicles than other existing approaches, while reducing the number of messages up to $46.73 \%$.
\end{abstract}

Keywords: Vehicular ad hoc networks, warning message dissemination, adverse density conditions, urban scenarios 


\section{Introduction}

Modern Intelligent Transportation Systems (ITS) are being propelled by the development and adoption of wireless telecommunications and computing technologies, thereby allowing our roads and highways to be both safer and more efficient transportation platforms.

Vehicular ad hoc Networks (VANETs) are wireless communication networks which support cooperative driving among vehicles on the road. Vehicles act as communication nodes and relays, forming dynamic vehicular networks together with other nearby vehicles (Ng and Waller, 2010; Santa et al., 2010). VANETs have particular features such as: distributed processing and organized networking, a large number of nodes, the distribution and the speed of these nodes, a constrained but highly variable network topology, variable communication conditions and mobility patterns, signal transmissions blocked by buildings, frequent partitioning due to the high mobility, and no significant power constraints.

The specific characteristics of VANETs favor the development of attractive and challenging services and applications, including road safety, traffic flow management, road status monitoring, environmental protection, and mobile infotainment (Jia et al., 2013; Paula et al., 2011; Zhou et al., 2011). In this work we focus on traffic safety and efficient warning message dissemination, where the main goal is to reduce the latency while increasing the accuracy of the information received by nearby vehicles when a dangerous situation occurs.

In a VANET, any vehicle detecting an abnormal situation on the road (i.e. accident, slippery road, etc.) starts notifying the anomaly to nearby vehicles to rapidly spread the information in a short period of time. Hence, broadcasting warning messages is of utmost importance to alert nearby vehicles. However, this dissemination is strongly affected by: (i) the signal attenuation due to the distance between the sender and receiver, (ii) the effect of obstacles on signal transmission (very usual in urban areas, e.g., due to buildings), and (iii) the instantaneous surrounding vehicle density.

Regarding (i) and (ii), the topology of the roadmap is an important factor that affects the average distance between the sender and the receiver, as well as the different obstacles present in the scenario. As for (iii), the warning message propagation scheme should be aware of the vehicle density, since 
lower densities can provoke message losses due to reduced communication capabilities, whereas higher densities may lead to reduced message delivery effectiveness due to serious redundancy, contention, and massive packet collisions caused by simultaneous forwarding, usually known as broadcast storm (Tseng et al., 2002).

So far, several authors have proposed different dissemination schemes to mitigate broadcast storms (Bi et al., 2010; N. Wisitpongphan et al., 2007; Soares et al., 2014; Suriyapaibonwattana and Pomavalai, 2008; Tseng et al., 2002). However, all of these schemes consider free space environments where no blocking obstacles are considered at all. They have not addressed the impact of buildings and other urban obstacles on the wireless signal propagation in realistic urban scenarios. The consequences derived from those incomplete analyses can be observed when their performance is tested in urban topologies, showing that they are unable to choose suitable relaying vehicles, or proving to be too restrictive to achieve an efficient message dissemination (Fogue et al., 2012b; Soares et al., 2011).

In this paper, we study the performance of typical broadcast dissemination schemes under hostile density conditions, i.e., vehicle densities that significantly differ from typical values in vehicular environments, and which are especially adverse for message dissemination. We consider that adapting the dissemination policy to the specific environment, accounting for the current vehicular density as well as for the scenario where the vehicles are located, can be beneficial in order to reduce broadcast storm related problems, and also to increase the efficiency of the warning message dissemination process. Based on this analysis, we propose both the Neighbor Store and Forward (NSF), scheme designed to be used under low density conditions, as well as the Nearest Junction Located (NJL) scheme, which was specially developed for high density conditions. Our main goal is to maximize the message delivery effectiveness, something difficult to achieve under adverse conditions.

The paper is organized as follows. Section 2 reviews existing dissemination schemes related to our proposal. In Section 3 we introduce our proposed schemes, i.e., the NSF and the NJL approaches. Section 4 shows the simulation environment used to validate our proposed algorithms. Section 5 presents and discusses the obtained results under very low and very high vehicle density scenarios. Finally, Section 6 presents the main conclusions drawn from this work. 


\section{Related Work}

Current research on vehicular networks usually focuses on analyzing scenarios representing common situations characterized by average density values. However, situations with very low or very high vehicle densities are often ignored, whereas they are very common in real vehicular environments. For example, outskirts or suburban areas usually experience density values below 25 vehicles $/ \mathrm{km}^{2}$, whereas traffic jams that appear in large cities present densities above 300 vehicles $/ \mathrm{km}^{2}$. We consider these scenarios as hostile conditions for vehicular networks, since the efficiency of warning message dissemination processes is noticeably reduced under these circumstances.

In this section we introduce some of the most relevant existing proposals related to message dissemination in vehicular networks. Before proposing new dissemination schemes specially suitable for adverse density conditions, we are going to analyze the performance of existing broadcast schemes for VANETs under these conditions, accounting for both low and high vehicle densities. Since the challenges to face in each situation are radically different, a separate study could be beneficial to maximize the performance of the message dissemination system.

\subsection{Low Density Conditions}

Vehicular scenarios including very low vehicle densities are frequently found in highways (Liu et al., 2012), and especially, in residential, rural, and outskirt traffic areas. The main goal when developing an emergency message dissemination system is to inform as many vehicles as possible in a short time period. Additionally, maintaining a low amount of wireless traffic is desirable to avoid the mentioned broadcast storm problem. When the density of vehicles is low, the relative importance of the number of messages received per vehicle is reduced, since the probability of overloading the channel with messages exchanged by just a few vehicles in minimal. Hence, suitable schemes for these situations should focus on forwarding warning messages in order to maximize message delivery, even when the probability of informing new vehicles is low. Some existing dissemination schemes that work more efficiently under low density conditions are the following:

- Flooding. This strategy is the simplest broadcast scheme, in which vehicles blindly rebroadcast every message they receive without applying additional control mechanisms. In low density scenarios, where the 
probability of broadcast storms is reduced, flooding represents a good candidate scheme.

- The Counter-based scheme (Tseng et al., 2002). Initially proposed for Mobile Ad Hoc Networks (MANETs), this scheme aims at mitigating broadcast storms by using a threshold $C$ and a counter $c$ to keep track of the number of times a broadcast message is received. Whenever $c \geq C$, rebroadcast is inhibited.

- The enhanced Street Broadcast Reduction (eSBR) (Martinez et al., 2010). This scheme is specially designed to be used in VANETs, taking advantage of the information provided by maps and built-in positioning systems, such as the Global Positioning System (GPS). Vehicles are only allowed to rebroadcast messages if they are located far from the sender vehicle, or if the vehicles are located in different streets, thereby providing access to new areas of the scenario. The eSBR scheme uses information about the roadmap to avoid blind areas due to the presence of urban structures blocking the radio signal.

- Ohta et al. (2011) proposed a new reliable data forwarding mechanism that combines Epidemic routing, as well as the positioning information and the moving direction of a node obtained from Global Positioning System (GPS), to reduce widespread forwarded data packets. The proposed scheme forwards data packets using the Store-Carry-Forward mechanism to the neighboring nodes that are determined by the positioning information and moving direction of a vehicle.

- More recently, Sou and Lee (2012) presented the Store-Carry-Broadcast (SCB) scheme. The main goal of this scheme is to assist message dissemination by broadcasting over a specific road segment instead of a single vehicle. In the SCB scheme, an opposite vehicle helps to disseminate the safety messages to oncoming vehicles traveling on the reverse lane by broadcasting. Compared with the well-known storecarry-forward scheme in VANETs, the SCB scheme consumes much less network bandwidth in terms of the number of broadcasts performed.

\subsection{High Density Conditions}

Another typical adverse scenario occurs when the vehicle density is above 300 vehicles $/ \mathrm{km}^{2}$, enough to produce traffic jams, or considerably reduce the 
speed of vehicles. This effect leads to an increase of the number of vehicles sending warning messages and beacons in a specific area, generating a likely scenario for channel contention and message collisions.

High Density situations usually require more restrictive dissemination schemes that allow reducing the number of messages sent, since the performance of the dissemination process may be highly reduced due to broadcast storms. Among the existing schemes that could face this effect we highlight the following:

- The Distance-based scheme (Tseng et al., 2002). This scheme accounts for the relative distance $d$ between vehicles to decide whether to rebroadcast a message. When the distance $d$ between two vehicles is short, the additional coverage area of the new rebroadcast is low, and so rebroadcasting the warning message is not recommended. Forwarding is only beneficial when the additional coverage is significant.

- Tseng et al. (2010) proposed a vehicle-density-based emergency broadcast (VDEB) scheme to solve the problem of high overhead in senderoriented schemes, and long delay in receiver-oriented schemes. Reducing the overhead could help reduce the broadcast storm problem in scenarios with a high vehicle density. However, the VDEB approach only works in one-dimensional scenarios, such as highways, and it is not useful in complex urban environments.

- The enhanced Message Dissemination for Roadmaps (eMDR) (Fogue et al., 2012b). As an improvement to the eSBR scheme, eMDR increases the efficiency of the system by avoiding multiple forwardings of the same message if nearby vehicles are located in different streets. Specifically, vehicles use the information about the junctions of the roadmap, and only the vehicle closest to the geographic center of the junction, according to the geopositioning system, is allowed to forward the messages received. This strategy aims at reducing the number of broadcasted messages while maintaining a high percentage of vehicles informed.

- Tonguz et al. (2010) presented the Distributed Vehicular Broad-CAST (DV-CAST) protocol. Specifically, DV-CAST is a distributed broadcast protocol that relies only on local topology information for handling broadcast messages in VANETs. DV-CAST handles the broadcast 
storm and the disconnected network problems simultaneously, while incurring a small amount of additional overhead. In particular, the DV-CAST protocol relies on local topology information (i.e., a list of one-hop neighbors) as the main criterion to determine how to handle message rebroadcasting, adapting the dissemination process depending on the density of neighbor vehicles, their position, and their direction.

\section{Novel Dissemination Schemes Proposed}

Due to the lack of dissemination schemes specifically designed for adverse density conditions, in this work we propose two different approaches specially designed for low and high density scenarios. The main objective is to achieve the highest percentage of informed vehicles in the shortest time possible. On the one hand, in environments with low vehicle densities, frequent network partitioning is a huge problem causing message losses and misinformation. On the other hand, in environments with high vehicle densities, the number of messages on the channel is a problem since they can provoke the well-known broadcast storm problem.

In low vehicle density scenarios, it is useful to store received messages until an optimal forwarding situation is found, instead of simply rebroadcasting messages at the time they are received, i.e., use a Store-and-Forward approach. According to this, we propose the Neighbor Store and Forward (NSF) scheme to improve the warning dissemination process in low density scenarios. This scheme requires the presence of a neighbor list in each of the vehicles, which is built using the one-hop beacons periodically exchanged by vehicles with information about their position and speed.

In addition, we propose the Nearest Junction Located (NJL), a scheme specially designed to reduce the broadcast storm problems in high density scenarios. Unlike NSF, this scheme does not require storing received warning messages or information about neighbors, since the high vehicle density is usually enough to provide good network connectivity. Instead, information about the road topology is used, and only vehicles placed at suitable locations are allowed to forward messages.

\subsection{Neighbor Store and Forward (NSF)}

In order to maximize the performance of the Story-Carry-Forward approach in sparse urban environments, we developed the NSF scheme. This scheme requires a neighbor list that is updated by means of one-hop beacons 
spread among vehicles, but instead of using information about the roadmap, NSF only relies on neighbor information.

We call neighbors those vehicles that are reachable by one-hop messages, without requiring any additional rebroadcast, i.e., they are within the communication range of the sender vehicle and the signal transmission is not blocked by any obstacle such as buildings. In our system, all the vehicles considered maintain a neighbor list that is built by using the beacons exchanged periodically among the nodes, avoiding any additional channel overhead. Whenever a new beacon is received, each vehicle checks its neighbor list to determine if the sender is a new neighbor, thereby adding this vehicle to the list. The neighbors' list is updated when new beacons are not received from a former neighbor after 2 seconds. In that case, the neighbor is removed from the list.

Figure 1 presents the flow chart of the Neighbor Store and Forward (NSF) scheme. In this scheme, after receiving a warning message, the vehicle stores it, and, before rebroadcasting the message, it checks if there are additional neighbor vehicles. Specifically, the vehicle waits to find a new neighbor to rebroadcast the message, i.e., until the vehicle receives a beacon from another vehicle which is not present in the neighbor list. The neighbor list is then updated, and stored messages are forwarded to inform new neighbors about the dangerous situation.

NSF is designed to inform new vehicles as they arrive to the affected area. Hence, the number of messages produced when the NSF scheme is used will be related to the number of vehicles in the scenario.

To better understand the operation of our proposed algorithm, we provide a formal definition of this dissemination scheme using set theory. In the mentioned formulation, the following notation is used:

- $\mathbb{V}$ : set of vehicles present in the scenario.

- $\mathbb{N}_{i}$ : set of neighbor vehicles of vehicle $v_{i} \in \mathbb{V}$.

- $\mathbb{S}_{i}$ : set of warning messages $m \in \mathbb{M}$ stored by vehicle $v_{i} \in \mathbb{V}$.

The formal definition of NSF requires some basic functions to express events and relationships between the components of the scenario. Specifically, the following functions are required: 


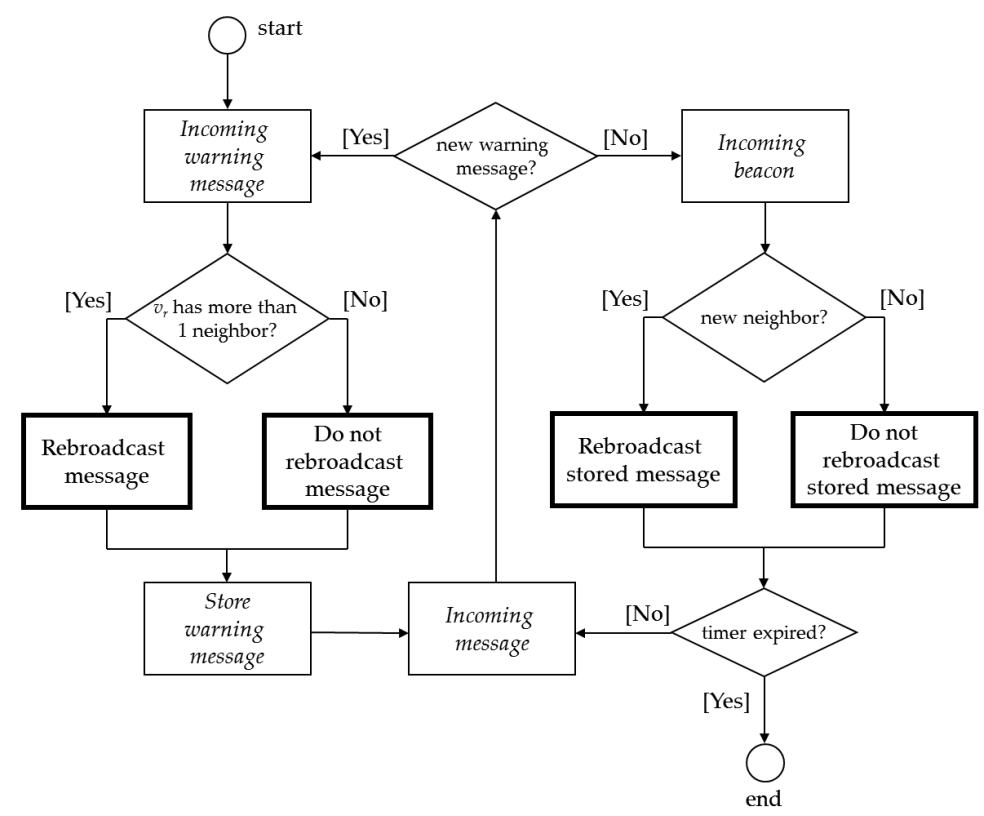

Figure 1: NSF dissemination scheme working flowchart.

- recv_beacon $\left(v_{r}, v_{s}, t\right)$ : vehicle $v_{r}$ receives a one-hop beacon from vehicle $v_{s}$ at time $t$.

- rebroadcast $(v, m, t)$ : vehicle $v$ broadcasts a warning message $m$ at time $t$.

Equation 1 contains the operation of the NSF algorithm after storing a warning message. As can be observed, NSF only rebroadcasts stored messages when a new vehicle is detected, i.e., a vehicle which is not currently included in the neighbor list.

$$
\begin{aligned}
& \forall v_{r} \in \mathbb{V} \wedge \exists v_{s} \in \mathbb{V} \wedge \text { recv_beacon }\left(v_{r}, v_{s}, t\right) \wedge \exists m \in \mathbb{S}_{r} \Rightarrow \\
& \left(\operatorname{rebroadcast}\left(v_{r}, m, t\right) \wedge \mathbb{N}_{r}=\mathbb{N}_{r} \cup\left\{v_{s}\right\} \Leftrightarrow v_{s} \notin \mathbb{N}_{r}\right)
\end{aligned}
$$

\subsection{Nearest Junction Located (NJL)}

Some dissemination schemes, such as eMDR, have proved to be effective at reducing broadcast storms in typical urban environments. However, the 
number of messages produced can be excessive in high vehicle density scenarios. Simpler schemes (i.e., that do not account for the effect of obstacles in signal propagation), such as the distance-based scheme offer a reduced number of messages but do not achieve optimal results in terms of informed vehicles in most scenarios. In addition, Store-and-Forward schemes are not usually necessary due to the lower frequency of partitions in highly congested networks (Fogue et al., 2012b).

To cope with these deficiencies, we propose a dissemination scheme called Nearest Junction Located (NJL). Unlike existing approaches, NJL is completely based on the topology of the roadmap where the vehicles are located, allowing vehicles to rebroadcast a message only if they are the nearest vehicle to the geographical coordinates of any junction obtained from the integrated maps, which we proved to be the most suitable location to access new areas of the topology. This scheme also requires maintaining a neighbor list in each vehicle to determine the relative position of the surrounding vehicles.

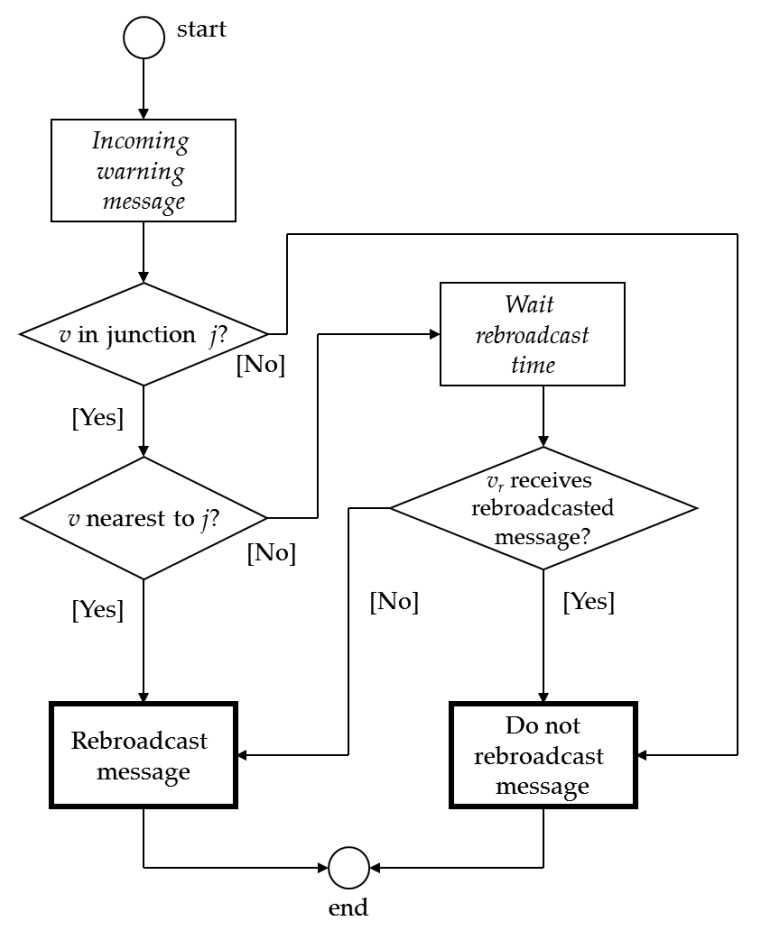

Figure 2: NJL scheme working flowchart. 
NJL only focuses on the location of the receiving vehicle, ignoring the distance between sender and receiver. Figure 2 shows the working flowchart of NJL. Whenever a vehicle receives a warning message, it determines whether it is the nearest one to any junction of the road layout by comparing its location to the locations of the neighbor vehicles. The scheme includes a security mechanism to avoid malfunction due to the radio interface or GPS errors, waiting for a rebroadcast backoff time before forwarding the message whenever a better positioned vehicle is expected (right side part of the flow chart).

Although the performance of this approach is not optimal in sparse environments due to its restrictiveness, it performs efficiently in high density scenarios where the dominant factor to improve the dissemination process is the position of the vehicles.

To better understand the operation of NJL, we provide a formal definition of this dissemination scheme using set theory. In the mentioned formulation, the following notation is used:

- $\mathbb{V}$ : set of vehicles present in the scenario.

- $\mathbb{M}$ : set of warning messages disseminated by vehicles.

- $\mathbb{J}$ : set of junctions of the road layout.

- $\mathbb{N}_{i}$ : set of neighbor vehicles of vehicle $v_{i} \in \mathbb{V}$.

The definition of NJL requires some basic functions to express events and relationships between the components of the scenario. Specifically, the following functions are required:

- $\operatorname{recv}\left(v_{r}, v_{s}, m, t\right)$ : vehicle $v_{r}$ receives a warning message $m$ from vehicle $v_{s}$ at time $t$.

- $\operatorname{dist}\left(e_{1}, e_{2}\right)$ : Euclidean distance between elements $e_{1}$ and $e_{2}$, i.e.:

$$
\operatorname{dist}\left(e_{1}, e_{2}\right)=\sqrt{\left(e_{1} \cdot x-e_{2} \cdot x\right)^{2}+\left(e_{1} \cdot y-e_{2} \cdot y\right)^{2}}
$$

- rebroadcast $(v, m, t)$ : vehicle $v$ broadcasts a warning message $m$ at time $t$. 
Equation 3 shows the formulation of the NJL algorithm using the notation previously introduced. As shown, NJL ignores the distance between sender and receiver and it only allows rebroadcasting if the receiver vehicle is the closest to the geographical center of the junction with respect to its neighbors.

$$
\begin{aligned}
& \forall v_{r} \in \mathbb{V} \wedge \exists m \in \mathbb{M}, v_{s} \in \mathbb{V} \wedge \operatorname{recv}\left(v_{r}, v_{s}, m, t\right) \Rightarrow \\
& \left(\operatorname { r e b r o a d c a s t } ( v _ { r } , m , t ) \Leftrightarrow \left(\exists j \in \mathbb{J} \wedge \operatorname{dist}\left(v_{r}, j\right)<t h_{j} \wedge\right.\right. \\
& \left.\left.\left(\nexists v_{n} \in \mathbb{N}_{r} \wedge \operatorname{dist}\left(v_{n}, j\right)<\operatorname{dist}\left(v_{r}, j\right)\right)\right)\right)
\end{aligned}
$$

\section{Simulation Environment}

To analyze and test our proposed broadcast methods we used the ns-2 simulator (Fall and Varadhan, 2000), modified to include the IEEE 802.11p standard $^{1}$. In terms of the physical layer, the data rate used for packet broadcasting is $6 \mathrm{Mbit} / \mathrm{s}$, as this is the maximum rate for broadcasting in 802.11p. The MAC layer was also extended to include four different channel access priorities. Therefore, application messages are categorized into four different Access Categories (ACs), where AC0 has the lowest and AC3 the highest priority.

The purpose of the $802.11 \mathrm{p}$ standard is to provide the minimum set of specifications required to ensure interoperability between wireless devices when attempting to communicate in potentially fast-changing communication environments. For our simulations, we chose the IEEE 802.11p because it is expected to be widely adopted by the industry.

The simulator was also modified to make use of our Real Attenuation and Visibility (RAV) scheme (Martinez et al., 2013), which proved to increase the level of realism in VANET simulations using real urban roadmaps in the presence of obstacles. The mobility of the vehicles was generated using CityMob for Roadmaps (C4R) (Fogue et al., 2012a), a mobility generator based on SUMO (Krajzewicz et al., 2012), able to import maps directly from OpenStreetMap (OpenStreetMap, 2012).

Figure 3 shows the topology used in our simulations, obtained from the downtown areas of the cities of Valencia (Spain) and San Francisco (USA).

\footnotetext{
${ }^{1}$ All these improvements and modifications are available in http://www.grc.upv.es/software/
} 


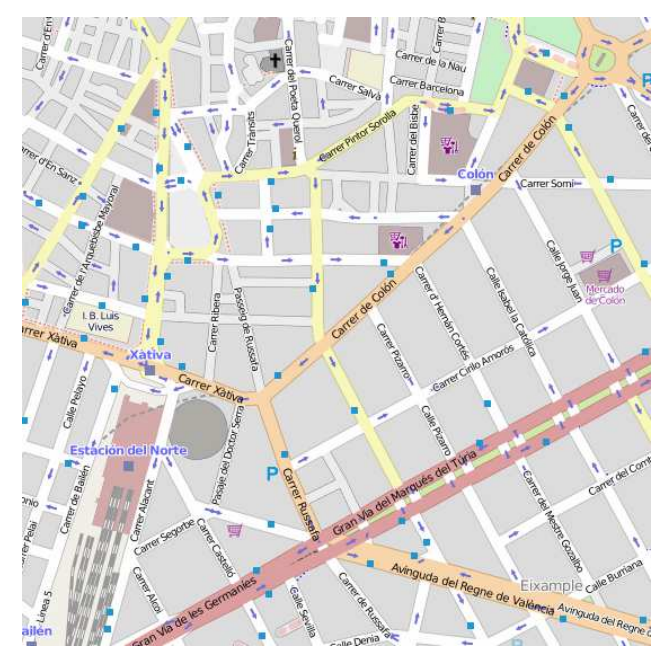

(a)

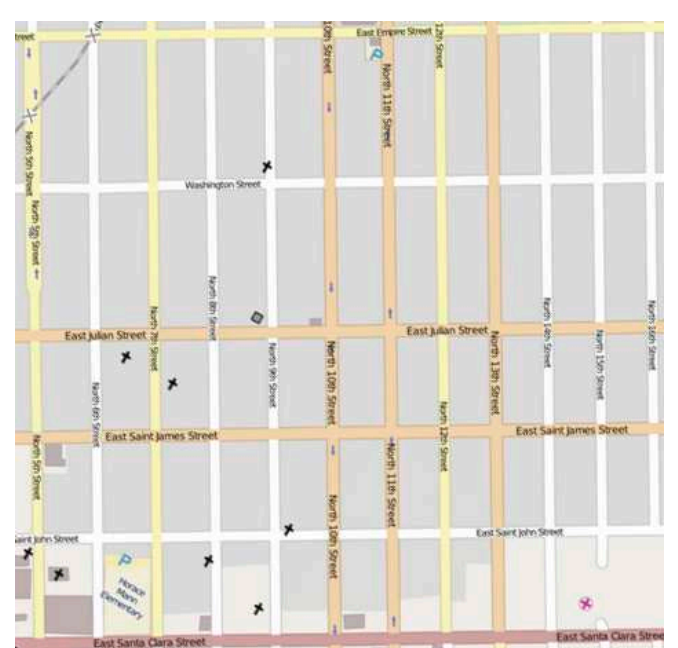

(b)

Figure 3: Maps of: (a) Valencia and (b) San Francisco used in the simulations.

The roadmaps used were selected in order to have different profile scenarios (i.e., with different topology characteristics). As shown in Figure 3 and according to (Sanguesa et al., 2013), we consider Valencia as a complex topology city, and San Francisco a simple topology city.

With regard to data traffic, vehicles operate in two modes: (a) warning mode, and (b) normal mode. Warning mode vehicles inform other vehicles about their status by sending warning messages periodically with the highest priority (AC3) at the MAC layer; each vehicle is only allowed to propagate them once for each sequence number. Normal mode vehicles enable the diffusion of these warning packets and, periodically, they also send beacons with information such as their positions, speed, etc. These periodic messages have lower priority $(\mathrm{AC} 1)$ than warning messages, and so they are not propagated by other vehicles. In our simulations, we included 1 warning mode vehicle in low density scenarios including 10,20 , and 30 vehicles $/ \mathrm{km}^{2}$, and 3 warning mode vehicles in high density scenarios accounting for 300,400 , and 500 vehicles $/ \mathrm{km}^{2}$. All the results represent an average of over 50 repetitions with different random scenarios, obtaining for all of them a confidence degree of 95\%. Table 1 shows the main parameters used for the simulations.

We are interested in the following performance metrics: (i) percentage of informed vehicles, (ii) number of messages received per vehicle, and (iii) 
Table 1: Parameter settings in the simulations.

\begin{tabular}{|l|c|}
\hline Parameter & Value \\
\hline \hline roadmap & Valencia and San Francisco \\
number of vehicles per km ${ }^{2}$ & {$[10,20,30,300,400$, and 500] } \\
number of collided vehicles & 1 and 3 \\
roadmap size & $1000 \mathrm{~m} \times 1000 \mathrm{~m}$ \\
warning message size & $256 B$ \\
beacon message size & $512 B$ \\
warning messages priority & $A C 3$ \\
beacon priority & $A C 1$ \\
interval between messages & 1 second \\
MAC/PHY & $802.11 \mathrm{p}$ \\
radio propagation model & RAV (Martinez et al., 2013) \\
mobility model & Krauss (Krauss et al., 1997) \\
channel bandwidth & $6 M b s$ \\
max. transmission range & $400 \mathrm{~m}$ \\
$d_{\text {min }}$ (used in distance-based, & $200 \mathrm{~m}$ \\
eSBR, and eMDR schemes) & \\
\hline
\end{tabular}

warning notification time. The percentage of informed vehicles is the percentage of vehicles that do receive the warning messages sent by warning mode vehicles. The number of packets received per vehicle (including beacons and warning messages) gives an estimation of channel contention, and of the overhead of the selected approach. Finally, the warning notification time is the time required by normal vehicles to receive a warning message sent by a warning mode vehicle.

\section{Simulation Results}

In this section we assess the performance of the proposed schemes (i.e., NSF and NJL) to prove their efficiency compared to existing mechanisms. From Section 2, we selected the flooding, counter-based, eSBR, and NSF schemes for performance comparison in those scenarios with very low densities, as well as the distance-based, eMDR, DV-CAST, and NJL schemes for performance comparison in very high density scenarios.

\subsection{Performance Evaluation in Low Vehicle Density Scenarios}

Figures 4 and 5 show the simulation results obtained when simulating the maps of Valencia and San Francisco with three different low vehicle densities: 10, 20, and 30 vehicles $/ \mathrm{km}^{2}$.

As shown, the different schemes provide similar results during the first 20 seconds of the simulation in terms of informed vehicles. However, after 


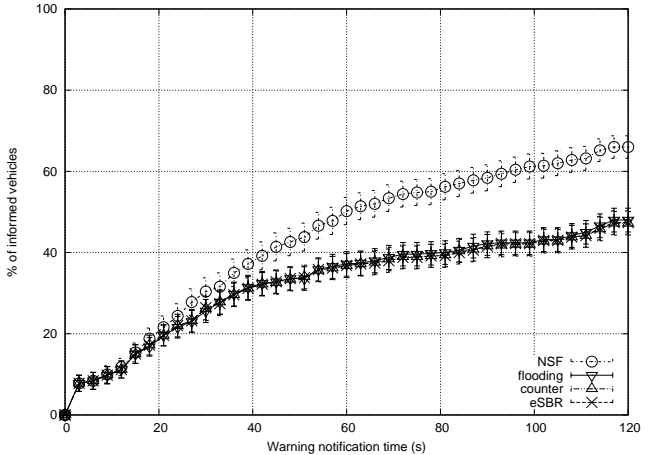

(a)

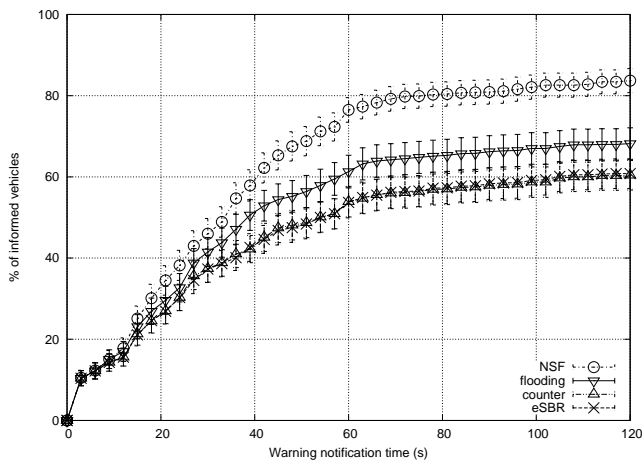

(b)

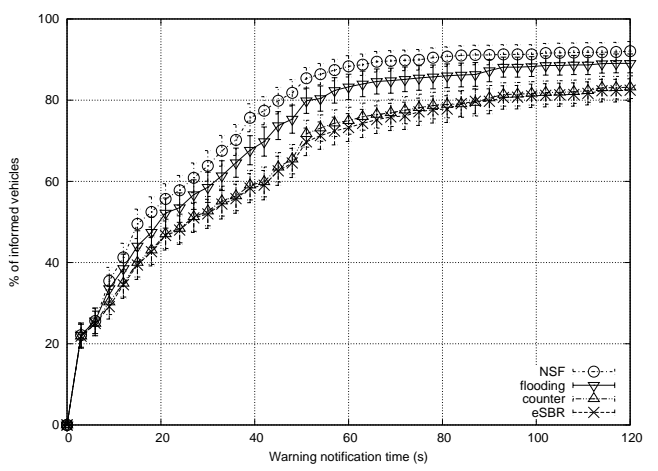

(c)

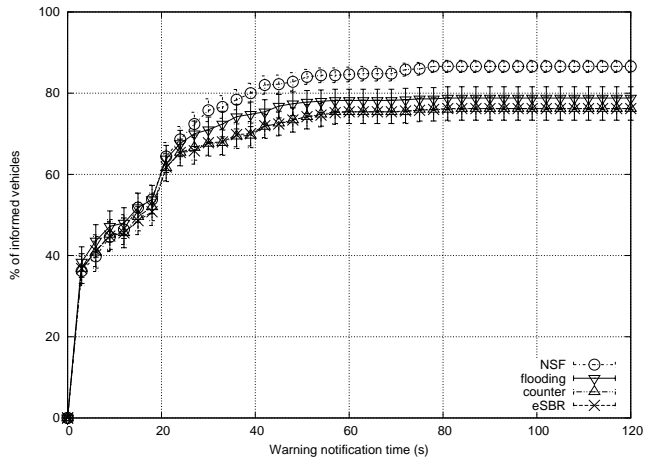

(d)

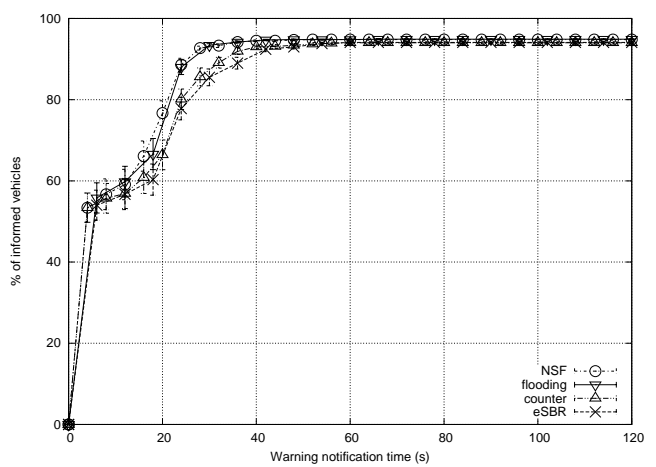

(e)

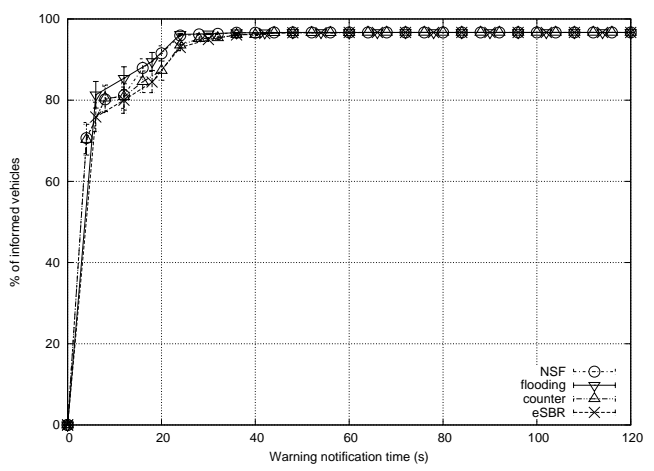

(f)

Figure 4: Percentage of informed vehicles in Valencia for: (a) 10, (b) 20, and (c) 30 vehicles $/ \mathrm{km} 2$, as well as in San Francisco for: (d) 10, (e) 20, and (f) 30 vehicles $/ \mathrm{km}^{2}$. 


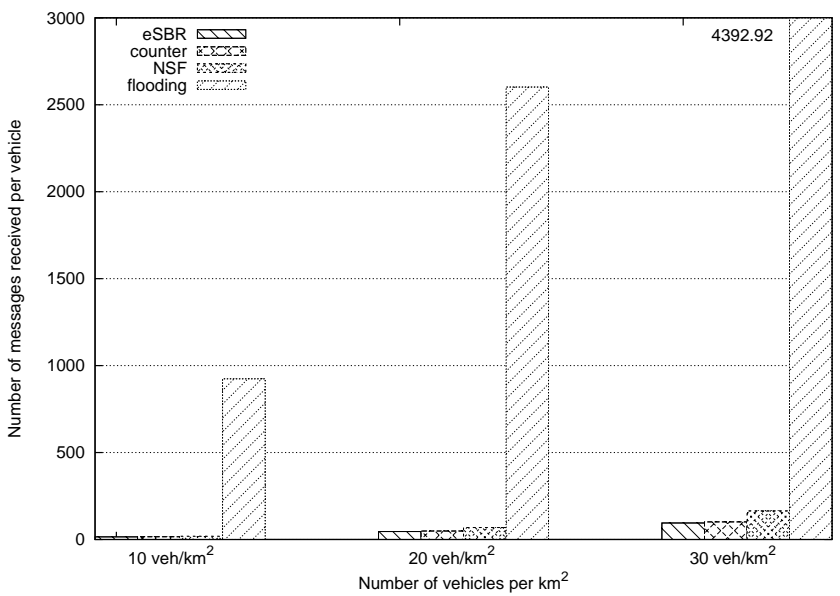

(a)

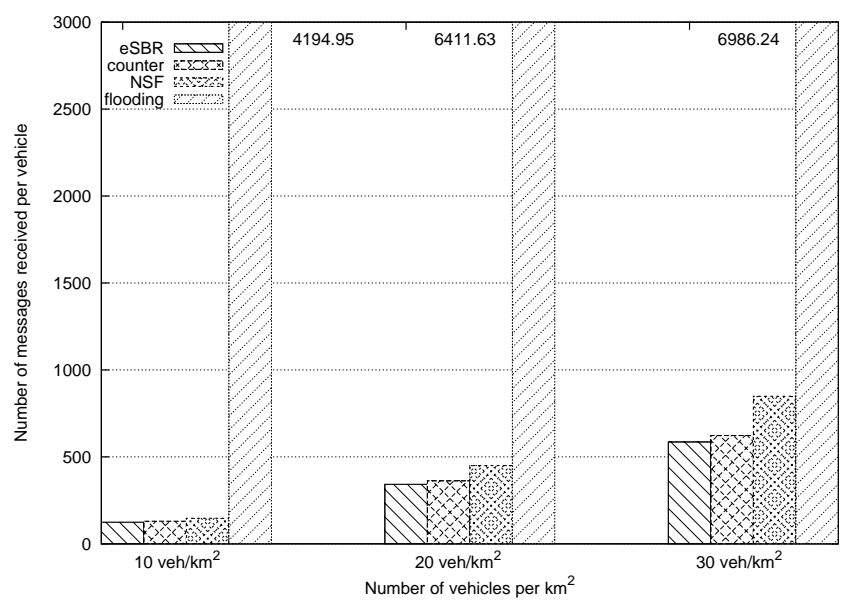

(b)

Figure 5: Number of messages received per vehicle under low vehicle density conditions in: (a) Valencia and (b) San Francisco. 
Table 2: Average time necessary to inform $60 \%$ of the vehicles

\begin{tabular}{|c|c|c|c|c|c|}
\hline Map & $\overline{\text { Density }}$ & NSF & Flooding & Counter & eSBR \\
\hline \multirow{3}{*}{ Valencia } & 10 veh. $/ \mathrm{km}^{2}$ & $\overline{95 \mathrm{~s}}$ & & & \\
\hline & 20 veh. $/ \mathrm{km}^{2}$ & $40 \mathrm{~s}$ & $58 \mathrm{~s}$ & $108 \mathrm{~s}$ & $105 \mathrm{~s}$ \\
\hline & 30 veh. $/ \mathrm{km}^{2}$ & $26 s$ & $32 \mathrm{~s}$ & $43 \mathrm{~s}$ & $43 s$ \\
\hline \multirow{3}{*}{ San Francisco } & 10 veh. $/ \mathrm{km}^{2}$ & $19 \mathrm{~s}$ & $20 \mathrm{~s}$ & $20 \mathrm{~s}$ & $20 \mathrm{~s}$ \\
\hline & 20 veh. $/ \mathrm{km}^{2}$ & $13 s$ & $13 s$ & $14 \mathrm{~s}$ & $17 \mathrm{~s}$ \\
\hline & 30 veh. $/ \mathrm{km}^{2}$ & $2 s$ & $2 s$ & $2 s$ & $2 s$ \\
\hline
\end{tabular}

the initial 20 seconds, the benefits of using a Store-and-Forward technique are especially noticeable. The NSF scheme informs more vehicles than the flooding, counter-based, and eSBR schemes while producing a similar number of messages (except for flooding, which significantly increases the number of messages received per vehicle). As an example, NSF is able to notify $83.7 \%$ of vehicles when simulating 20 vehicles $/ \mathrm{km}^{2}$ in the roadmap of Valencia after 120 seconds, whereas the eSBR and counter-based schemes only notify $60.8 \%$ and $60.4 \%$ of the vehicles, respectively, during the same period, requiring only a slight increase of the messages produced. The downside of the flooding scheme is that it requires an enormous amount of messages to inform only $68.2 \%$ of vehicles in the same scenario.

Focusing on the number of messages, and excluding the flooding approach, the differences between schemes are not significant due to the low chance of causing channel overload. The NSF scheme requires $54.27 \%$ more messages than the rest of schemes, on average, when simulating 30 vehicles $/ \mathrm{km}^{2}$, the additional overhead, in absolute terms, is very small. Moreover, the low vehicle density of the scenarios reduces the overall traffic in the wireless channel, and the effect on performance is not noticeable. Note that the number of messages received per vehicle is higher in San Francisco, since its topology is simpler, and warning messages can reach the rest of vehicles more easily.

Table 2 shows the average time required by the NSF, flooding, counterbased, and eSBR schemes to inform $60 \%$ of the vehicles in the scenario. As shown, the eSBR and counter-based schemes are $170 \%$ and $162.5 \%$ slower, respectively, when simulating Valencia under 20 vehicles $/ \mathrm{km}^{2}$, and $65.38 \%$ slower when simulating 30 vehicles $/ \mathrm{km}^{2}$ compared to NSF, in spite of the low differences in terms of number of messages received per vehicle. Regarding simpler maps like San Francisco, there are no significant differences between 
the schemes, especially for densities of 30 vehicles $/ \mathrm{km}^{2}$.

Finally, it is noticeable how the proposed NSF scheme is able to outperform the flooding scheme in terms of percentage of informed vehicles, while drastically reducing the number of messages received per vehicle. Hence, using Store-and-Forward strategies allows achieving better performance compared to existing dissemination schemes, obtaining significant improvements with a reduced amount of additional messages.

\subsection{Performance Evaluation in High Vehicle Density Scenarios}

Figures 6 and 7 show the results obtained in Valencia and San Francisco when simulating very high vehicle densities, i.e., 300, 400, and 500 vehicles $/ \mathrm{km}^{2}$.

As shown, the distance-based scheme offers a poor performance in terms of percentage of informed vehicles when compared to the NJL, DV-CAST, and eMDR dissemination schemes. Hence, it is not suitable for highly congested urban scenarios where warning message dissemination requires fast notification of dangerous situations. Note that the NJL, DV-CAST and eMDR schemes basically present the same results in terms of notification time and percentage of informed vehicles, whereas the number of messages received per vehicle when using the NJL scheme is reduced, ranging from $24.85 \%$ to $30.69 \%$ compared to eMDR, and from $43.51 \%$ to $44.09 \%$ compared to DV-CAST in Valencia, therefore making NJL the most suitable dissemination scheme in this kind of scenarios.

Regarding simple maps like San Francisco, since they have long and straight streets, channel contention and message collisions due to the higher number of vehicles in line-of-sight are prone to occur. In this kind of scenarios, NJL is again the scheme that provides the lower amount of messages, offering a reduction ranging from $40.94 \%$ to $46.73 \%$ compared to eMDR, from $32.84 \%$ to $44.01 \%$ compared to DV-CAST, and from $39.68 \%$ to $41.36 \%$ compared to the distance-based approach; the differences between the four schemes in terms of informed vehicles through time are null.

Since broadcast storms are prone to occur in high density situations due to serious redundancy, contention, and massive packet collisions caused by simultaneous forwarding, existing broadcast storm reduction techniques usually adopt very restrictive dissemination schemes that can compromise the reliability of the communication system. 


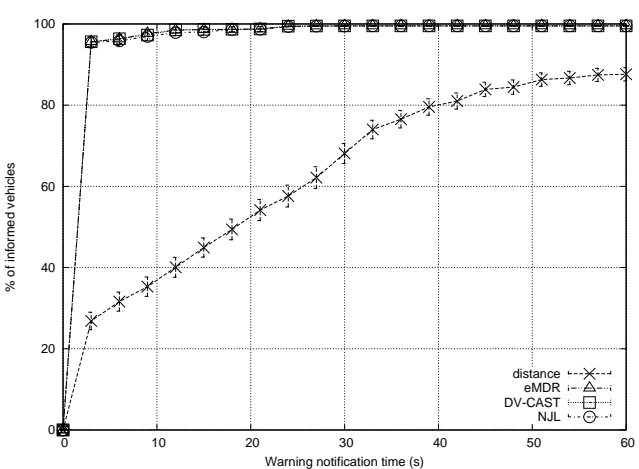

(a)

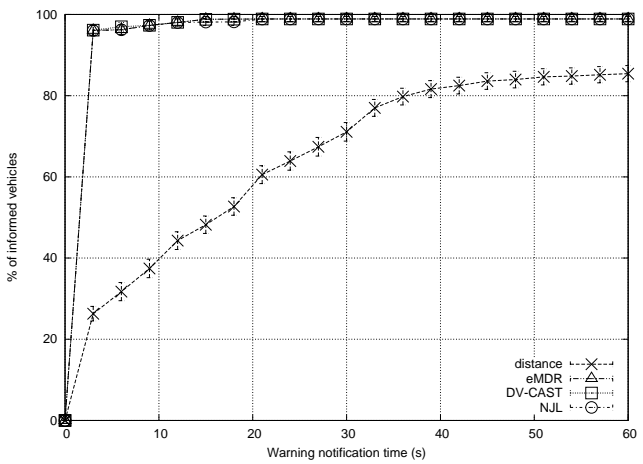

(b)

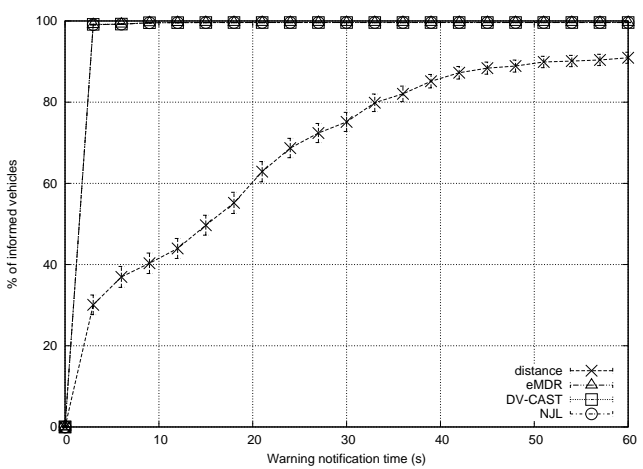

(c)

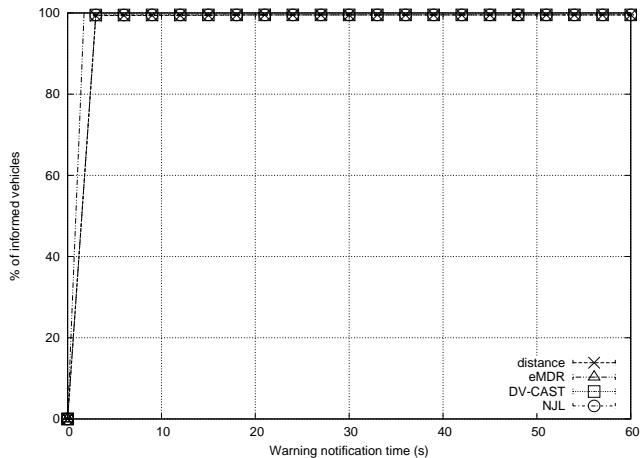

(d)

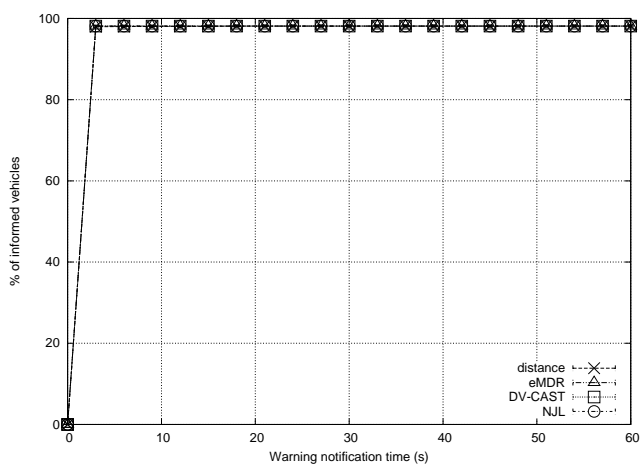

(e)

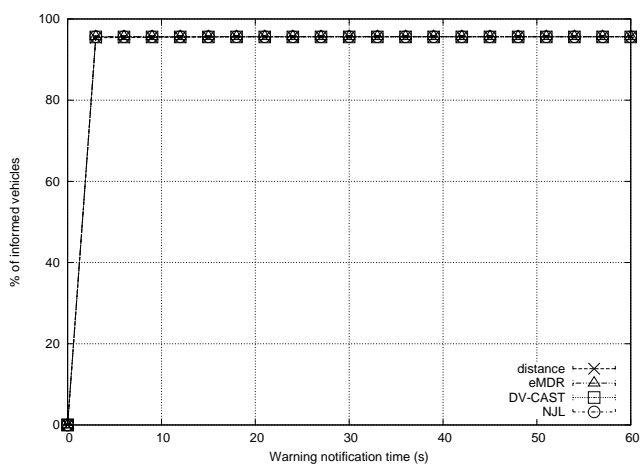

(f)

Figure 6: Percentage of informed vehicles in Valencia for: (a) 300, (b) 400, and (c) 500 vehicles $/ \mathrm{km}^{2}$, as well as in San Francisco for: (d) 300, (e) 400, and (f) 500 vehicles $/ \mathrm{km}^{2}$. 


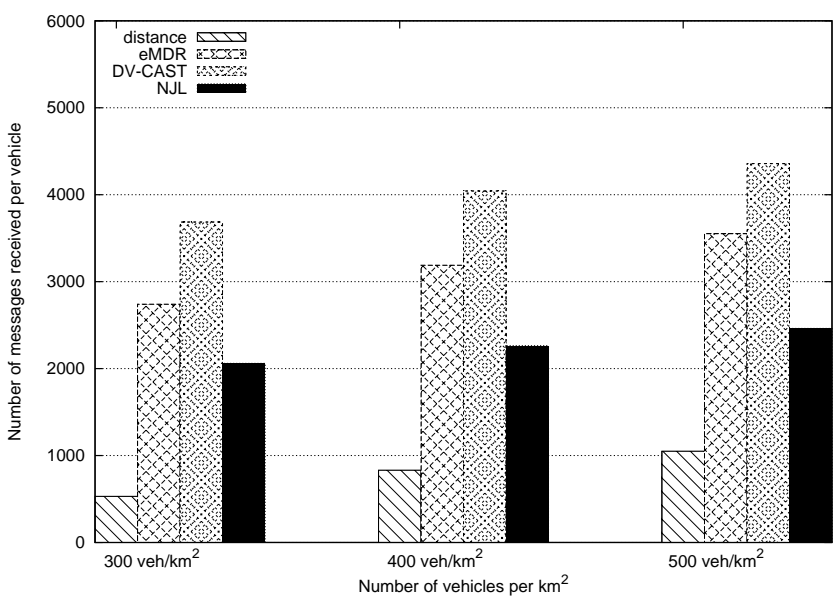

(a)

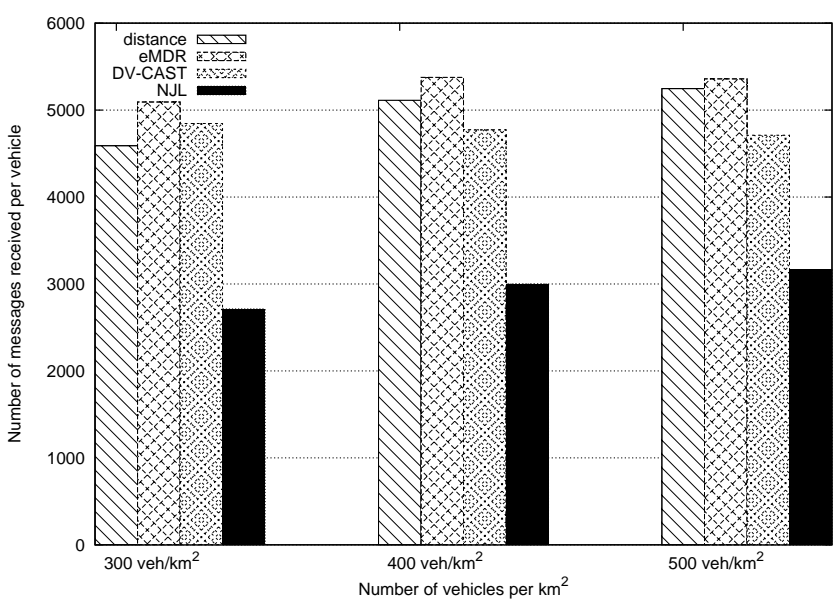

(b)

Figure 7: Number of messages received per vehicle under high vehicle density conditions in: (a) Valencia, and (b) San Francisco. 
Table 3: Performance of the different dissemination schemes under high density conditions.

\begin{tabular}{|c|c|c|c|c||c|c|c|}
\hline \multirow{3}{*}{ vehicles } & & \multicolumn{3}{|c||}{ Valencia } & \multicolumn{3}{|c|}{ San Francisco } \\
\cline { 2 - 8 } & Bcast & $P_{\text {Inf }}$ & $M_{\text {recv }}$ & efficiency & $P_{\text {Inf }}$ & $M_{\text {recv }}$ & efficiency \\
\hline \hline \multirow{4}{*}{300} & distance & $91.36 \%$ & 530.7 & 94.19 & $99.53 \%$ & 4589.98 & 53.88 \\
\cline { 2 - 8 } & eMDR & $99.56 \%$ & 2741.35 & 72.47 & $99.53 \%$ & 5093.06 & 48.83 \\
\cline { 2 - 8 } & DV-Cast & $99.56 \%$ & 3684.68 & 62.99 & $99.53 \%$ & 4845.73 & 51.31 \\
\cline { 2 - 8 } & NJL & $99.56 \%$ & 2060.03 & 79.31 & $99.53 \%$ & 2712.69 & 72.75 \\
\hline \hline \multirow{4}{*}{400} & distance & $89.17 \%$ & 831.37 & 90.68 & $98.13 \%$ & 5112.89 & 47.90 \\
\cline { 2 - 8 } & eMDR & $98.91 \%$ & 3188.47 & 67.76 & $98.13 \%$ & 5476.30 & 45.21 \\
\cline { 2 - 8 } & DV-Cast & $98.91 \%$ & 4041.61 & 59.14 & $98.13 \%$ & 4771.53 & 51.38 \\
\cline { 2 - 8 } & NJL & $98.91 \%$ & 2263.02 & 77.12 & $98.13 \%$ & 2998.38 & 69.45 \\
\hline \hline \multirow{4}{*}{500} & distance & $92.93 \%$ & 1050.21 & 88.70 & $95.57 \%$ & 5245.92 & 45.11 \\
\cline { 2 - 8 } & eMDR & $99.62 \%$ & 3551.35 & 64.35 & $95.57 \%$ & 5357.97 & 43.94 \\
\cline { 2 - 8 } & DV-Cast & $99.62 \%$ & 4357.68 & 56.26 & $95.57 \%$ & 4711.70 & 50.70 \\
\cline { 2 - 8 } & NJL & $99.62 \%$ & 2461.49 & 75.29 & $95.57 \%$ & 3164.20 & 66.89 \\
\hline
\end{tabular}

Table 3 shows the simulation results of different schemes in high density environments in terms of percentage of informed vehicles, and the average messages received per vehicle after 120 seconds. Additionally, we added a new metric called "efficiency" which gives an idea of the number of messages needed to inform $1 \%$ of vehicles (i.e., $\left.100-\left(M_{\text {recv }} / P_{\text {inf }}\right)\right)$. As shown, in simple maps such as San Francisco, all dissemination schemes offer similar results in terms of informed vehicles; however, in terms of efficiency NJL obtains better results, since it is able to reduce the number of messages needed to inform the same percentage of vehicles. As for complex maps such as Valencia, where the number of junctions is higher and the distance of streets is shorter, although the distance-based scheme offers the best results in terms of efficiency, it is not able to achieve the same percentage of informed vehicles, making it unreliable in the warning dissemination context. Once the distance-based approach is discarded, the NJL is the scheme which offers higher efficiency, reducing significantly the number of messages needed to inform the same percentage of vehicles.

Overall, and according to results obtained, the proposed NJL scheme is the most suitable dissemination mechanism to be used in both simple and complex maps under very high vehicle density environments, significantly reducing the number of messages required to inform the same percentage of vehicles compared to other schemes. NJL mitigates the broadcast storm problem without compromising the reliability of the system. 


\section{Conclusions}

In this paper we studied the performance of different warning message dissemination schemes for VANETs under situations classified as adverse due to the very low or very high density of vehicles in the scenario. The efficiency of warning message dissemination processes under these conditions is reduced as a result of frequent network partitioning under low densities, and high channel contention under high vehicle densities. In particular, we proposed two dissemination approaches specially designed for these situations: the Neighbor Store and Forward (NSF) scheme for very low vehicle densities, and the Nearest Junction Located (NJL) scheme for very high vehicle densities.

Simulation results showed that our proposed schemes outperform the existing dissemination algorithms in terms of informed vehicles and messages received per vehicle. Comparing its performance with the flooding, counterbased, and eMDR schemes, the NSF scheme allowed reducing the warning notification time in low vehicle density scenarios, while increasing up to $23.3 \%$ the percentage of informed vehicles. As for high vehicle density conditions, the proposed NJL scheme proved to be the most efficient of the tested schemes, being able to inform the same percentage of vehicles than other existing approaches (i.e., distance-based, eMDR and DV-CAST), while reducing the number of messages up to $46.73 \%$.

\section{Acknowledgments}

This work was partially supported by the Ministerio de Ciencia e Innovación, Spain, under Grant TIN2011-27543-C03-01, by the Fundación Universitaria Antonio Gargallo and the Obra Social de Ibercaja, under Grant 2013/B010, as well as the Government of Aragón and the European Social Fund (T91 Research Group).

\section{References}

Bi, Y., Cai, L., Shen, X., Zhao, H., May 2010. A Cross Layer Broadcast Protocol for Multihop Emergency Message Dissemination in Inter-Vehicle Communication. In: IEEE International Conference on Communications (ICC). pp. 1-5.

Fall, K., Varadhan, K., February 2000. ns notes and documents. The VINT Project. UC Berkeley, LBL, USC/ISI, and Xerox PARC, available at http://www.isi.edu/nsnam/ns/ns-documentation.html. 
Fogue, M., Garrido, P., Martinez, F. J., Cano, J.-C., Calafate, C. T., Manzoni, P., March 2012a. A Realistic Simulation Framework for Vehicular Networks. In: 5th International ICST Conference on Simulation Tools and Techniques (SIMUTools 2012), Desenzano, Italy. pp. 37-46.

Fogue, M., Garrido, P., Martinez, F. J., Cano, J.-C., Calafate, C. T., Manzoni, P., December 2012b. Evaluating the impact of a novel message dissemination scheme for vehicular networks using real maps. Transportation Research Part C: Emerging Technologies 25, 61-80.

Jia, D., Lu, K., Wang, J., 2013. On the network connectivity of platoonbased vehicular cyber-physical systems. Transportation Research Part C: Emerging Technologies (0), - .

Krajzewicz, D., Erdmann, J., Behrisch, M., Bieker, L., December 2012. Recent development and applications of SUMO - Simulation of Urban MObility. International Journal On Advances in Systems and Measurements 5 (3\&4), 128-138.

Krauss, S., Wagner, P., Gawron, C., 1997. Metastable states in a microscopic model of traffic flow. Physical Review E 55 (5), 5597-5602.

Liu, B., Khorashadi, B., Ghosal, D., Chuah, C.-N., Zhang, H. M., 2012. Analysis of the information storage capability of VANET for highway and city traffic. Transportation Research Part C: Emerging Technologies 23 (0), $68-84$.

Martinez, F. J., Fogue, M., Coll, M., Cano, J.-C., Calafate, C., Manzoni, P., 2010. Evaluating the impact of a novel warning message dissemination scheme for VANETs using real city maps. In: Crovella, M., Feeney, L., Rubenstein, D., Raghavan, S. (Eds.), NETWORKING 2010. Vol. 6091 of Lecture Notes in Computer Science. Springer Berlin / Heidelberg, pp. 265-276.

Martinez, F. J., Fogue, M., Toh, C. K., Cano, J.-C., Calafate, C. T., Manzoni, P., 2013. Computer simulations of VANETs using realistic city topologies. Wireless Personal Communications 69 (2), 639-663.

N. Wisitpongphan, O.K. Tonguz, J.S. Parikh, P. Mudalige, F. Bai, V. Sadekar, 2007. Broadcast storm mitigation techniques in vehicular ad hoc networks. IEEE Wireless Communications 14, 84-94. 
Ng, M., Waller, S. T., 2010. A static network level model for the information propagation in vehicular ad hoc networks. Transportation Research Part C: Emerging Technologies 18 (3), 393-407, 11th IFAC Symposium: The Role of Control.

Ohta, Y., Ohta, T., Kohno, E., Kakuda, Y., 2011. A Store-Carry-ForwardBased Data Transfer Scheme Using Positions and Moving Direction of Vehicles for VANETs. In: 10th International Symposium on Autonomous Decentralized Systems (ISADS). pp. 131-138.

OpenStreetMap, 2012. Collaborative project to create a free editable map of the world. Available at http://www.openstreetmap.org.

Paula, M., Isento, J., Dias, J., Rodrigues, J., June 2011. A real-world VDTN testbed for advanced vehicular services and applications. In: IEEE 16th International Workshop on Computer Aided Modeling and Design of Communication Links and Networks (CAMAD). pp. 16-20.

Sanguesa, J. A., Fogue, M., Garrido, P., Martinez, F. J., Cano, J.-C., Calafate, C. T., Manzoni, P., November 2013. On the selection of optimal broadcast schemes in VANETs. In: The 16th ACM/IEEE International Conference on Modeling, Analysis and Simulation of Wireless and Mobile Systems (MSWIM). Barcelona, Spain, pp. 411-418.

Santa, J., Toledo-Moreo, R., Zamora-Izquierdo, M. A., Ubeda, B., GomezSkarmeta, A. F., 2010. An analysis of communication and navigation issues in collision avoidance support systems. Transportation Research Part C: Emerging Technologies 18 (3), 351-366.

Soares, V., Farahmand, F., Rodrigues, J., 2011. Traffic differentiation support in vehicular delay-tolerant networks. Telecommunication Systems 48 (1-2), 151-162.

Soares, V. N., Rodrigues, J. J., Farahmand, F., 2014. Geospray: A geographic routing protocol for vehicular delay-tolerant networks. Information Fusion 15 (0), 102 - 113, special Issue: Resource Constrained Networks.

Sou, S.-I., Lee, Y., 2012. SCB: Store-Carry-Broadcast Scheme for Message Dissemination in Sparse VANET. In: IEEE 75th Vehicular Technology Conference (VTC Spring). pp. 1-5. 
Suriyapaibonwattana, K., Pomavalai, C., October 2008. An effective safety alert broadcast algorithm for VANET. In: International Symposium on Communications and Information Technologies (ISCIT). pp. 247-250.

Tonguz, O. K., Wisitpongphan, N., Bai, F., 2010. DV-CAST: A distributed vehicular broadcast protocol for vehicular ad hoc networks. IEEE Wireless Communications 17 (2), 47-57.

Tseng, Y.-C., Ni, S.-Y., Chen, Y.-S., Sheu, J.-P., 2002. The broadcast storm problem in a mobile ad hoc network. Wireless Networks 8, 153-167.

Tseng, Y.-T., Jan, R.-H., Chen, C., Wang, C.-F., Li, H.-H., 2010. A vehicledensity-based forwarding scheme for emergency message broadcasts in VANETs. In: IEEE 7th International Conference on Mobile Adhoc and Sensor Systems (MASS). pp. 703-708.

Zhou, L., Zhang, Y., Song, K., Jing, W., Vasilakos, A., Feb 2011. Distributed media services in p2p-based vehicular networks. IEEE Transactions on Vehicular Technology 60 (2), 692-703. 